\title{
Profiling changes in microRNAs of immature dendritic cells differentiated from human monocytes
}

\author{
MENGYU SUN, JINGYI WU, WENTIAN LIU \\ Department of Gastroenterology and Hepatology, Tianjin Medical University General Hospital, Tianjin, China
}

\begin{abstract}
MicroRNAs (miRNAs) critically impact a wide array of eukaryotic developmental and physiologic processes through post-transcriptional gene silencing. In this study, we employed miRNA array and investigated in vitro the miRNA profile of immature dendritic cells (iDCs) derived from monocytes isolated from human venous blood. Our results showed that there were 379 miRNAs which were detectable in both monocytes and iDCs among the 856 miRNAs assayed, of which 155 miRNAs were detectable in monocytes while 224 miRNAs were detectable in iDCs. There were 103 miRNAs differentially expressed which could be relevant to the differentiation of iDCs from human monocytes. Sixty-two out of 103 miRNAs were upregulated whereas 41 miRNAs were downregulated. Of particular interest were the tremendous upregulation of miR122a and the downregulation of miR200c in iDCs. In addition, it was found that the strikingly downregulated miRNAs in iDCs also included miR-335, miR-514, miR-509, miR-31, miR-442b, miR-1, miR-199a, miR-203, miR-363 and miR-489 whereas the upregulation of miR-210, miR-155, miR-126, miR-139, miR-452, miR-19a, miR-25 and miR-181d were remarkable. Our results revealed a profile change of miRNAs when human iDCs were differentiated from monocytes as a result of in vitro stimulation with relevant cytokines.
\end{abstract}

Key words: microRNA, dendritic cell, monocyte, microarray.

(Cent Eur J Immunol 2021; 46 (1): 10-16)

\section{Introduction}

Human monocytes, macrophages and dendritic cells (DCs) constitute a compartment that initiates the first line of defense against microbial invasion by the regulation of innate and adaptive immune responses [1,2]. The mobilization and differentiation from monocytes to immature dendritic cells (iDCs) is one of the key processes involved in the homeostasis of the immune system. A particular stimulus or a combination such as interleukin (IL)-6, interferon $\gamma$ (IFN- $\gamma$ ), granulocyte-macrophage colony-stimulating factor (GM-CSF), IL-4 or microbial pattern-associated agents can drive the differentiation of iDCs to a particular subset of DC which enhances or suppresses the immune response $[3,4]$. Mature DCs are fully activated DCs that deliver activating signals to T cells by the production of IL-12, IL-6, and IFN- $\gamma$ and the expression of co-stimulating molecules such as CD86 and CD80. In contrast, tolerogenic DCs could also be induced from immature DCs to negatively regulate the immune response by induction of regulatory T cells and induction of $\mathrm{T}$ cell anergy, production of immunosuppressive molecules such as IL-10, IL-27, nitric oxide, etc. [1, 5].

MicroRNAs (miRNAs) are highly conserved, small non-coding RNA molecules containing approximately
22 nucleotides that regulate gene expression by binding to 3'-UTR regions of mRNA. These molecules are essential in cell differentiations, cell-cell interactions and cellular responses to stimuli. In the immune system, miRNAs play crucial roles in regulating the activation and differentiation of immune cells and consequently modulate cytokine release, antibody production, etc. [6, 7]. Previous studies showed that miRNAs regulate the functions of DCs through signaling mediators such as p27kip1, KPC1, and SOCS-1, or tumor-derived exosomes [8, 9]. MiR-155 and miR-142 were reported to be involved in the modulation of DC maturation [8-10]. Analysis of individual miRNAs in terms of immune cells was studied by previous research groups [6-14]; however, overall analyses of miRNAs using microarray could display the comprehensive expression pattern of miRNA and facilitate further investigation on the regulation of immune responses by miRNA.

This study aimed to depict the profile of miRNA in human monocytes and iDCs and to identify the key miRNAs that are involved in the differentiation of iDCs. Human monocytes were cultured in vitro and induced by IL-4 plus GM-CSF to differentiate into DCs. MiRNA microarray chips were utilized to analyze the changes of the expression of miRNAs in both monocytes and iDCs. The results

Correspondence: Wentian Liu, Department of Gastroenterology and Hepatology, Tianjin Medical University General Hospital, Tianjin, China, e-mail: 1wentian64@163.com

Submitted: 7.09.2019; Accepted: 2.01.2020 
showed that miR-122a and miR200c exhibited the most striking changes involved in the differentiation of iDCs. The results of this study help understand the regulation of iDC development by miRNA.

\section{Material and methods}

\section{Study participants}

The peripheral blood samples were collected from healthy volunteers $(n=5)$ recruited in Tianjin Medical University General Hospital. Written, informed consent was obtained from all participating individuals before the procedure. The study was approved by the Ethical Committee of Tianjin Medical University General Hospital.

\section{Differentiation of monocytes into iDCs}

For iDC generation, monocytes were isolated by adhesion from Ficoll gradient-separated peripheral blood mononuclear cells (PBMCs). The cell preparation was tested for purity by flow cytometry. Monocytes were identified as CD14(+) HLA-DR(+) cells. The purity from this purification was in excess of $90 \%$. Monocytes were subjected to cytokine-driven differentiation into iDCs by 6-day culture in the presence of GM-CSF and IL-4, and iDC cell differentiation was evaluated by flow cytometry on day 6 . Differentiated iDCs were defined as DCSIGN(+) CD1a(+) CD206(+) HLA-DR(high) CD86(high) CD14(-) and CD15(-) cells. iDC yields following culture were $>95 \%$.

\section{Microarray profiling of miRNAs}

Microarray analysis of 856 miRNAs was carried out by $\mu$ Paraflo technology (LC Sciences, TX, USA). Data signals were collected by an Axon GenePix 4000B Microarray Scanner. A total of 856 human mature miRNA are listed in the Sanger miRNA Sequence Database (miRBase Release 12.0). Total RNAs were extracted from the adherent monocytic fraction $\left(10^{7}\right.$ cells) or cytokine differentiated iDCs $(n=5)$ using a mirVana miRNA Isolation kit (Ambion, TX, USA) according to the manufacturer's instructions. The miRNA samples were label-blinded and subjected to array hybridization. The miRNAs that differed quantitatively (at least 2-fold) from counterpart monocytes in differential subtraction analysis were considered to be remarkably changed miRNAs illustrating that DC differentiation was accompanied by definable changes in miRNA expression. miRNAs that displayed profound differential expression (at least 4-fold) were selected for confirmatory analysis by real-time PCR analysis using pre-validated miRNA-specific primers (Applied Biosystems, Foster City, CA).

\section{Real-time PCR analysis}

MiRNAs were extracted using a mirVana miRNA Isolation kit (Ambion, TX, USA). cDNAs were synthesized by a miRScript II RT kit (Qiagen) according to the manu- facturer's instructions. The expression of miRNA was determined using the TaqMan MicroRNA assays (Applied Biosystems) according to the manufacturer's protocol. miRNA expression was normalized to RNU43, a small nuclear RNA endogenous control. Expression levels of the following miRNAs were assayed: miR200c, miR335, miR514, miR509, miR31, miR442b, miR1, miR199a, miR203, miR363, miR489 miR122a, miR210, miR155, miR126, miR139, miR452, miR19a, miR25 and miR181d. The expression data were analyzed using the $2^{-\Delta \Delta C T}$ method.

\section{Statistical analysis}

For microarray data analysis, the ANOVA test was used to evaluate the differences between monocytes and iDCs. When the differences of expression were $\log 2$ fold-change $>2$ or $\log 2$ fold-change $<-2$, it is considered meaningful. Student's $t$ test was applied to compare the differences in the expression of miRNAs between the monocyte group and iDC group measured by real-time PCR. Statistical significance was set at two-tailed $p<0.05$.

\section{Results}

\section{Detectable miRNAs expressed in human monocytes and iDCs with miRNA microarray}

Human monocytes were isolated from peripheral venous blood and cultured in the presence of IL-4 and GMCSF to drive their differentiation to DCs. Cells from five donors were prepared and analyzed by miRNA microarray which contains 856 miRNAs spotted on the chip. The microarray detected a total of 381 miRNAs in which

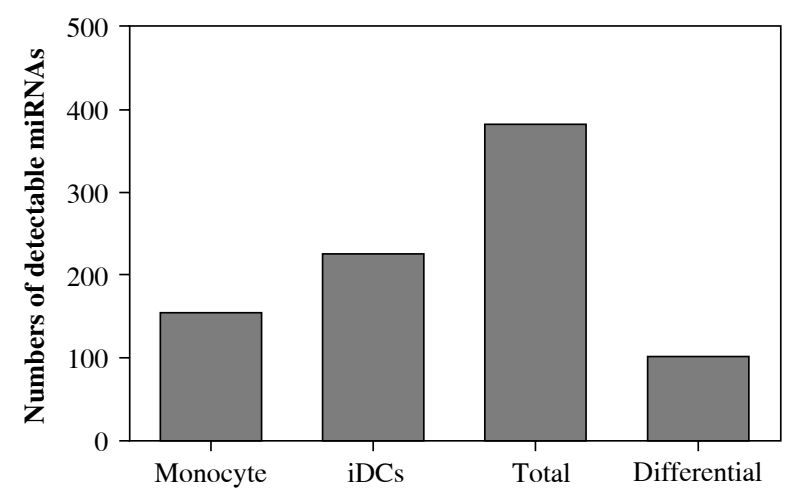

Fig. 1. Numbers of detectable miRNAs in the experimental groups analyzed by miRNA microarray. Human monocytes were differentiated to dendritic cells (DCs) in culture with IL-4 and GM-CSF. MiRNAs extracted from monocytes and iDCs $(n=5)$ were subject to microarray hybridization in a blinded manner. MiRNAs were considered detectable when both $\mathrm{Cy} 3$ and $\mathrm{Cy} 5$ signals were reported by the instrument. Differential expression was defined by at least twofold changes in signal intensity in iDCs when compared to that in monocyte control in differential subtraction analysis 

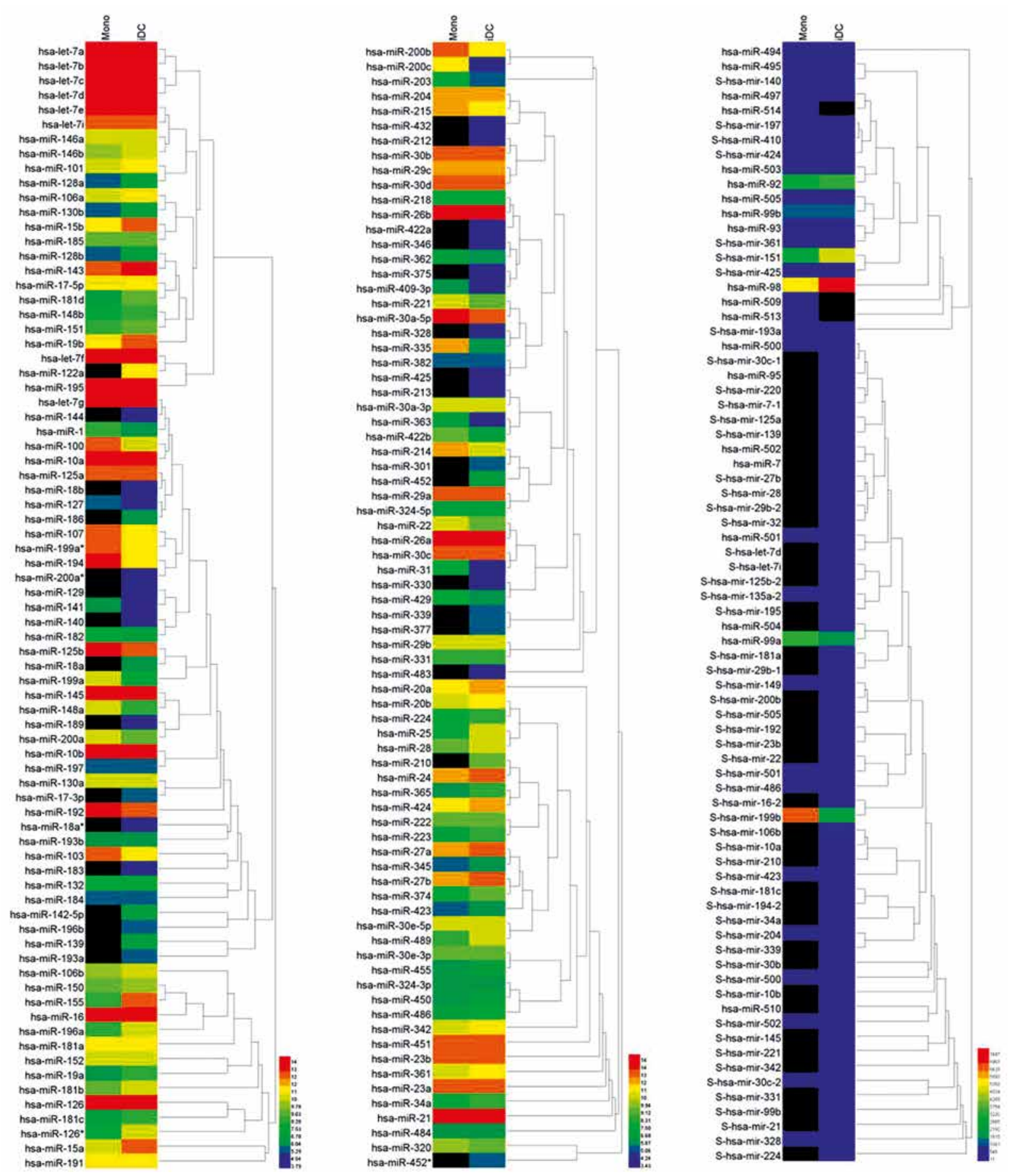

Fig. 2. Heatmap illustration of miRNAs expressed in iDCs and the original control monocytes. MiRNAs extracted from both monocytes and iDCs were subject to microarray hybridization containing 587 miRNAs spotted on the chip. Detectable miRNAs were plotted on a heatmap using Heml v1.0 software. Black color refers to undetectable signals which were recorded as 0 as digitized signals. Red color indicates the highest expression and blue color indicates the lowest expression

152 miRNAs were present in both monocytes and iDCs. There were 3 miRNAs which were detectable only in monocytes whereas 74 miRNAs were present only in iDCs. There were 103 miRNAs differentially expressed between monocytes and iDCs (Fig. 1). The fluorescence signals represent- ing miRNA expression were plotted as a heatmap and all detectable miRNAs were listed (Fig. 2). The results demonstrated that much more miRNAs were expressed in iDCs than in monocytes, implying that miRNA might play a more important role in keeping biological function of iDCs. 
Table 1. Differential expression of miRNAs in iDCs derived from human monocytes

\begin{tabular}{|c|c|c|c|c|c|c|c|c|}
\hline MicroRNAs & $\begin{array}{c}\text { Down- } \\
\text { regulated }\end{array}$ & $\begin{array}{c}\text { Up- } \\
\text { regulated }\end{array}$ & MicroRNAs & $\begin{array}{c}\text { Down- } \\
\text { regulated }\end{array}$ & $\begin{array}{c}\text { Up- } \\
\text { regulated }\end{array}$ & MicroRNAs & $\begin{array}{l}\text { Down- } \\
\text { regulated }\end{array}$ & $\begin{array}{c}\text { Up- } \\
\text { regulated }\end{array}$ \\
\hline hsa-miR-200c & -6.98 & & hsa-miR-15b & & 1.40 & hsa-miR-30e-3p & & 0.57 \\
\hline hsa-miR-122a & & 6.00 & hsa-miR-181c & & 1.39 & hsa-miR-451 & & 0.57 \\
\hline hsa-miR-335 & -5.84 & & hsa-miR-194 & -1.29 & & hsa-miR-204 & -0.55 & \\
\hline hsa-miR-514 & -4.12 & & hsa-miR-342 & & 1.28 & hsa-miR-150 & & 0.50 \\
\hline hsa-miR-509 & -4.08 & & hsa-miR-500 & -1.17 & & hsa-miR-221 & -0.50 & \\
\hline hsa-miR-210 & & 4.01 & hsa-miR-200a & -1.16 & & hsa-miR-30c & -0.48 & \\
\hline hsa-miR-155 & & 3.77 & hsa-miR-99a & -1.13 & & hsa-miR-10a & -0.48 & \\
\hline hsa-miR-31 & -3.51 & & hsa-miR-20b & & 1.09 & hsa-miR-98 & & 0.48 \\
\hline hsa-miR-126 & & 3.13 & hsa-miR-224 & & 1.08 & hsa-miR-361 & & 0.48 \\
\hline hsa-miR-139 & & 3.06 & hsa-miR-181b & & 1.03 & hsa-miR-126 & & 0.45 \\
\hline hsa-miR-452 & & 2.78 & hsa-miR-107 & -1.03 & & hsa-miR-92 & & 0.39 \\
\hline hsa-miR-422b & -2.75 & & hsa-miR-22 & -1.02 & & hsa-miR-195 & & 0.39 \\
\hline hsa-miR-19a & & 2.71 & hsa-miR-125b & -0.98 & & hsa-miR-424 & & 0.38 \\
\hline hsa-miR-1 & -2.54 & & hsa-miR-103 & -0.97 & & hsa-miR-26b & -0.36 & \\
\hline hsa-miR-199a & -2.44 & & hsa-miR-27a & & 0.91 & hsa-miR-29c & -0.33 & \\
\hline hsa-miR-25 & & 2.34 & hsa-miR-146b & & 0.85 & hsa-let-7e & & 0.32 \\
\hline hsa-miR-181d & & 2.30 & hsa-miR-148b & & 0.84 & hsa-miR-29a & -0.31 & \\
\hline hsa-miR-203 & -2.20 & & hsa-miR-101 & & 0.83 & hsa-miR-30b & -0.31 & \\
\hline hsa-miR-363 & -2.17 & & hsa-miR-10b & -0.78 & & hsa-miR-23a & & 0.30 \\
\hline hsa-miR-489 & & 2.02 & hsa-miR-20a & & 0.77 & hsa-let-7a & & 0.28 \\
\hline hsa-miR-196a & & 1.98 & hsa-let-7i & & 0.76 & hsa-let-7b & & 0.26 \\
\hline hsa-miR-365 & & 1.80 & hsa-miR-17-5p & & 0.76 & hsa-miR-23b & & 0.23 \\
\hline hsa-miR-148a & -1.72 & & hsa-miR-19b & & 0.75 & hsa-let-7d & & 0.20 \\
\hline hsa-miR-429 & -1.71 & & hsa-miR-27b & & 0.74 & hsa-let-7c & & 0.19 \\
\hline hsa-miR-214 & -1.69 & & hsa-miR-181a & & 0.70 & hsa-miR-24 & & 0.18 \\
\hline hsa-miR-223 & & 1.67 & hsa-miR-143 & & 0.68 & hsa-let-7f & & 0.15 \\
\hline hsa-miR-15a & & 1.64 & hsa-miR-106b & & 0.68 & S-hsa-mir-486 & -2.63 & \\
\hline hsa-miR-28 & & 1.57 & hsa-miR-30d & -0.68 & & S-hsa-mir-224 & & 2.46 \\
\hline hsa-miR-374 & & 1.56 & hsa-miR-192 & -0.67 & & S-hsa-mir-149 & -1.64 & \\
\hline hsa-miR-21 & & 1.49 & hsa-miR-146a & & 0.63 & S-hsa-mir-423 & -1.51 & \\
\hline hsa-miR-200b & -1.49 & & hsa-miR-93 & & 0.63 & S-hsa-mir-199b & -1.37 & \\
\hline hsa-miR-199a & -1.48 & & hsa-miR-151 & & 0.63 & S-hsa-mir-151 & & 0.65 \\
\hline hsa-miR-34a & & 1.45 & hsa-miR-30a-5p & -0.63 & & S-hsa-mir-140 & & 0.57 \\
\hline hsa-miR-100 & -1.41 & & hsa-miR-106a & & 0.61 & & & \\
\hline hsa-miR-455 & & 1.40 & hsa-miR-16 & & 0.57 & & & \\
\hline
\end{tabular}

\section{Differential expression of miRNAs between human monocytes and induced iDCs}

It is of interest to note that there were a number of profoundly altered miRNAs involved in the differentiation of iDCs from monocytes. One hundred and three miRNAs out of 381 detectable miRNAs in both monocytes and iDCs were differentially expressed miRNAs (Table 1). MiR-122a and miR-210 were markedly upregulated whereas miR-509, miR-514, miR-335 and miR-200c were downregulated at the cutoff line of 4-fold change (Fig. 3). There were 20 miRNAs that exhibited differential expres- 


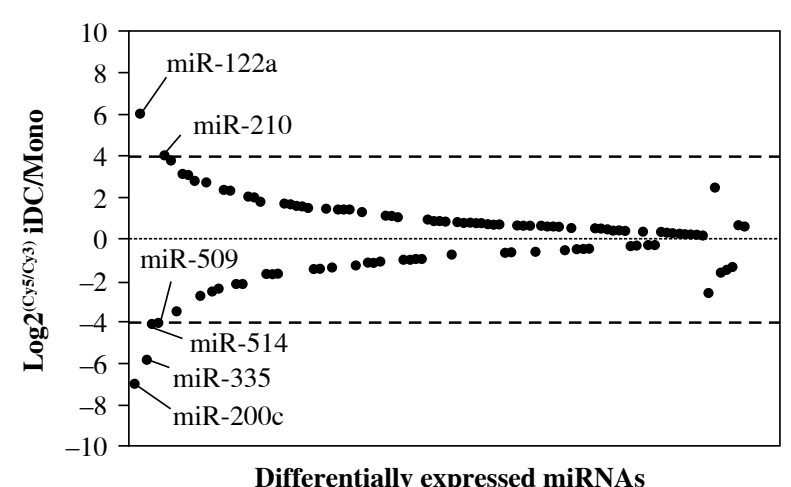

Fig. 3. Differential expression of miRNAs in monocytes and derived iDCs. The differentially expressed miRNAs were defined as at least 2-fold for $\log 2^{(\mathrm{Cy} 5 / \mathrm{Cy} 3)}$ for the ratio of expression in iDCs to monocytes. The $\mathrm{Y}$-axis indicates the ratio of miRNA expression. The $\mathrm{X}$-axis indicates each individual of differentially expressed miRNA. When the ratio was beyond 4-fold changes, the names of those miRNA were labeled in the plot. These miRNAs were selected for confirmatory analyses using real-time PCR

sion levels when the cutoff line was set at 2-fold change. In this regard, the upregulated miRNAs included miR-31, miR-442b, miR-1, miR-199a, miR-203, miR-363 and miR-489 whereas the upregulation of miR-155, miR-126, miR-139, miR-452, miR-19a, miR-25 and miR-181d was considerable. The results provided an overview of the miRNA profile implicated in the differentiation of iDCs from monocytes in humans.
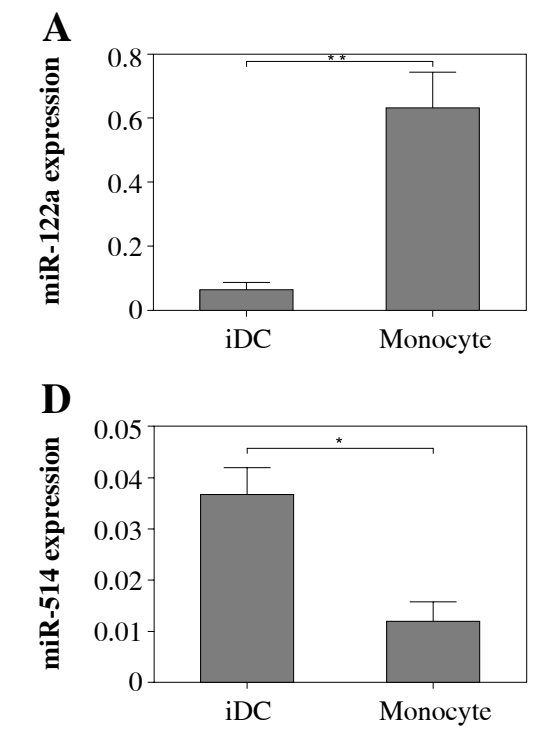

\section{Analysis of expression of miRNAs by real-time PCR}

To confirm the expression of those miRNA with pronounced difference in monocytes and iDCs, we utilized real-time PCR to determine the expression of six individual miRNAs selected with at least 4-fold alteration between monocytes and differentiated DCs. The expression of both miR-122a and miR-210 was significantly elevated in iDCs as compared to monocytes. In contrast, the expression levels of miR-509, miR-514, miR-335 and miR-200c were found to be decreased significantly in iDCs as compared to monocytes (Fig. 4). The results of real-time PCR were consistent with the data generated by miRNA microarray.

\section{Discussion}

The miRNA system is a complex network that largely silences or degrades the transcripts of mRNA by being incorporated into Argonaute (Ago) protein within the RNA-induced silencing complex [15-21]. In developmental biology, miRNAs have long been known to be involved in cell differentiation and organ development [22]. Previous efforts have been made to understand the regulation of immune cells by miRNA. In T cell and B cell developmental and differentiation, several miRNAs were reported to play important roles in the differentiation of $\mathrm{T}$ cells such as miR-150, miR155, miR-181, miR-17-92; B cells such as miR-101, miR-152, miR-155, miR-181, miR-17-92; and Tregs such as miR-146, miR-150, miR1-55 [23-36]. In the innate immune system, miRNA contributes to the maturation of DCs. miR155 was induced by activating the NF- $\kappa \mathrm{B}$

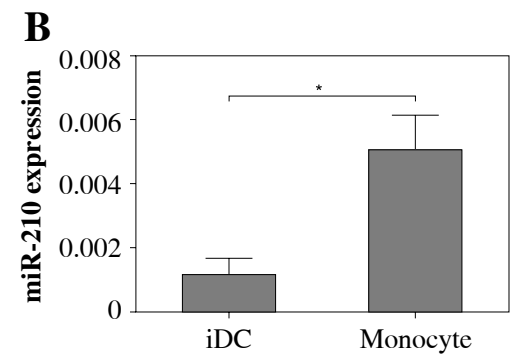

$\mathbf{E}$

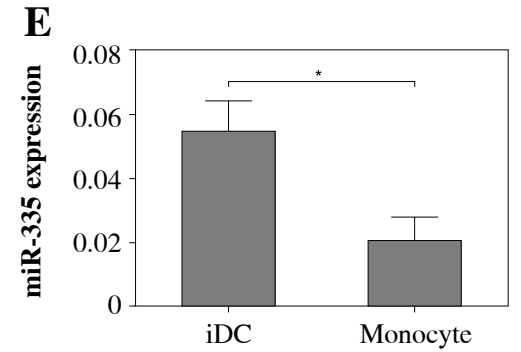

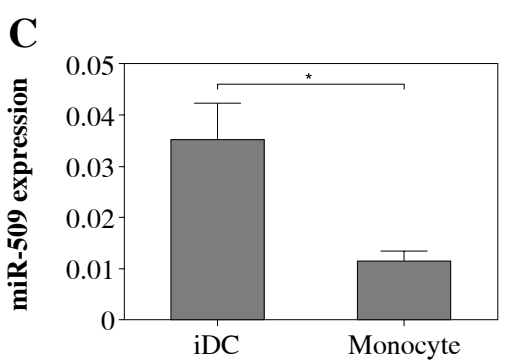

$\mathrm{F}$

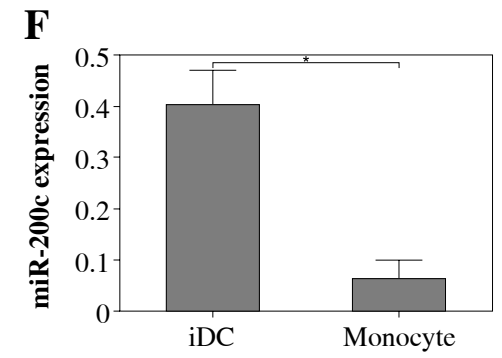

Fig. 4. Real-time PCR analysis of the expression of selected miRNAs. The expression of selected miRNAs was determined using real-time PCR. MiRNA expression was normalized to RNU43 small nuclear RNA endogenous controls. The relative expression of miRNA was calculated using the $2^{-\Delta \Delta C T}$ method. The data were expressed as mean \pm standard deviation of multiplicate wells. Asterisks refer to $p<0.05$ calculated using Student's $t$ test 
and Jun kinase pathway and targeted SOCS1 to regulate the maturation of DCs [7]. miR146a was involved in the TLR signaling cascade in DC maturation [37]. miR155 was considered to be an important miRNA that regulates the immune cells as evident by these previous reports. In our study, the data showed that miR155 was also markedly upregulated in iDC (the value of $\log 2^{\text {(Cy5/Cy3) }}$ was 3.77 ), as seen in Table 1 . In addition to the known miRNAs reported elsewhere, our results revealed that miR122a was most strikingly upregulated in iDCs derived from human monocytes upon GM-CSF and IL-4 stimulation in vitro.

In this study, we found that the expression of miR200c was the lowest among all decreased miRNAs in iDCs. miR200c was reported to be associated with the growth of tumors $[38,39]$. Another downregulated miRNA, miR335, was also found to be related to the pathogenesis of bladder, prostate and breast cancers [40-42]. The downstream targets of miR335 could be multiple events such as ROCK1, NO synthase, etc. The results revealed several important miRNAs implicated in the cytokine-induced differentiation from monocytes to iDCs.

\section{Acknowledgments}

We would like to thank the volunteers and donors of Tianjin Medical University General Hospital.

The authors declare no conflict of interest.

\section{References}

1. Geissmann F, Manz MG, Jung S, et al. (2010): Development of monocytes, macrophages, and dendritic cells. Science 327 : 656-661.

2. Bieber K, Autenrieth SE (2015): Insights how monocytes and dendritic cells contribute and regulate immune defense against microbial pathogens. Immunobiology 220: 215-226.

3. Felzmann T, Hüttner KG, Breuer SK, et al. (2005): Semi-mature IL-12 secreting dendritic cells present exogenous antigen to trigger cytolytic immune responses. Cancer Immunol Immunother 54: 769-780.

4. Vopenkova K, Mollova K, Buresova I, Michalek J (2012): Complex evaluation of human monocyte-derived dendritic cells for cancer immunotherapy. J Cell Mol Med 16: 28272837.

5. Banchereau J, Briere F, Caux C, et al. (2000): Immunobiology of dendritic cells. Annu Rev Immunol 18: 767-811.

6. Liston A, Linterman M, Lu LF (2010): MicroRNA in the adaptive immune system, in sickness and in health. J Clin Immunol 30: 339-346.

7. Lu LF, Liston A (2009): MicroRNA in the immune system, microRNA as an immune system. Immunology 127: 291-298.

8. Taghikhani A, Hassan ZM, Ebrahimi M, Moazzeni SM (2019): microRNA modified tumor-derived exosomes as novel tools for maturation of dendritic cells. J Cell Physiol 234: 9417-9427.

9. Lu C, Huang X, Zhang X, et al. (2011): miR-221 and miR155 regulate human dendritic cell development, apoptosis, and IL-12 production through targeting of p27kip1, KPC1, and SOCS-1. Blood 117: 4293-4303.

10. Wang Y, Liang J, Qin H, et al. (2016): Elevated expression of miR-142-3p is related to the pro-inflammatory function of monocyte-derived dendritic cells in SLE. Arthritis Res Ther 18: 263.

11. Hashimi ST, Fulcher JA, Chang MH, et al. (2009): MicroRNA profiling identifies miR-34a and miR-21 and their target genes JAG1 and WNT1 in the coordinate regulation of dendritic cell differentiation. Blood 114: 404-414.

12. Kuipers H, Schnorfeil FM, Brocker T (2010): Differentially expressed microRNAs regulate plasmacytoid vs. conventional dendritic cell development. Mol Immunol 48: 333-340.

13. Stumpfova Z, Hezova R, Meli AC, et al. (2014): MicroRNA profiling of activated and tolerogenic human dendritic cells. Mediators Inflamm 2014: 259689.

14. Tserel L, Runnel T, Kisand K, et al. (2011): MicroRNA expression profiles of human blood monocyte-derived dendritic cells and macrophages reveal miR-511 as putative positive regulator of Toll-like receptor 4. J Biol Chem 286: 26487-26495.

15. Grishok A, Pasquinelli AE, Conte D, et al. (2001): Genes and mechanisms related to RNA interference regulate expression of the small temporal RNAs that control C. elegans developmental timing. Cell 106: 23-34.

16. Hutvágner G, McLachlan J, Pasquinelli AE, et al. (2001): A cellular function for the RNA-interference enzyme Dicer in the maturation of the let-7 small temporal RNA. Science 293: 834-838

17. Hutvágner G, Zamore PD (2002): A microRNA in a multiple-turnover RNAi enzyme complex. Science 297: 20562060.

18. Ketting RF, Fischer SE, Bernstein E, et al. (2001): Dicer functions in RNA interference and in synthesis of small RNA involved in developmental timing in C. elegans. Genes Dev 15: 2654-2659.

19. Lee Y, Jeon K, Lee JT, et al. (2002): MicroRNA maturation: stepwise processing and subcellular localization. EMBO J 21: 4663-4670.

20. Lingel A, Simon B, Izaurralde E, Sattler M (2003): Structure and nucleic-acid binding of the Drosophila Argonaute 2 PAZ domain. Nature 426: 465-469.

21. Mourelatos Z, Dostie J, Paushkin S, et al. (2002): miRNPs: a novel class of ribonucleoproteins containing numerous microRNAs. Genes Dev 16: 720-728.

22. Bartel DP, Chen CZ (2004): Micromanagers of gene expression: the potentially widespread influence of metazoan microRNAs. Nat Rev Genet 5: 396-400.

23. Chen CZ, Li L, Lodish HF, Bartel DP (2004): MicroRNAs modulate hematopoietic lineage differentiation. Science 303: 83-86.

24. Cobb BS, Hertweck A, Smith J, et al. (2006): A role for Dicer in immune regulation. J Exp Med 203: 2519-2527.

25. Li QJ, Chau J, Ebert PJ, et al. (2007): miR-181a is an intrinsic modulator of T cell sensitivity and selection. Cell 129: 147-161.

26. Lu LF, Thai TH, Calado DP, et al. (2009): Foxp3-dependent microRNA155 confers competitive fitness to regulatory T cells by targeting SOCS1 protein. Immunity 30: 80-91.

27. Marson A, Kretschmer K, Frampton GM, et al. (2007): Foxp3 occupancy and regulation of key target genes during T-cell stimulation. Nature 445: 931-935.

28. Rodriguez A, Vigorito E, Clare S, et al. (2007): Requirement of bic/microRNA-155 for normal immune function. Science 316: 608-611. 
29. Teng G, Hakimpour P, Landgraf P, et al. (2008): MicroRNA-155 is a negative regulator of activation-induced cytidine deaminase. Immunity 28: 621-629.

30. Thai TH, Calado DP, Casola S, et al. (2007): Regulation of the germinal center response by microRNA-155. Science 316 : 604-608.

31. Ventura A, Young AG, Winslow MM, et al. (2008): Targeted deletion reveals essential and overlapping functions of the miR-17 through 92 family of miRNA clusters. Cell 132: 875-886.

32. Vigorito E, Perks KL, Abreu-Goodger C, et al. (2007): microRNA-155 regulates the generation of immunoglobulin class-switched plasma cells. Immunity 27: 847-859.

33. Xiao C, Calado DP, Galler G, et al. (2007): MiR-150 controls B cell differentiation by targeting the transcription factor c-Myb. Cell 131: 146-159.

34. Xiao C, Srinivasan L, Calado DP, et al. (2008): Lymphoproliferative disease and autoimmunity in mice with increased miR-17-92 expression in lymphocytes. Nat Immunol 9: 405414.

35. Zheng Y, Josefowicz SZ, Kas A, et al. (2007): Genome-wide analysis of Foxp3 target genes in developing and mature regulatory T cells. Nature 445: 936-940.

36. Zhou B, Wang S, Mayr C, et al. (2007): miR-150, a microRNA expressed in mature B and T cells, blocks early B cell development when expressed prematurely. Proc Natl Acad Sci U S A 104: 7080-7085.

37. Taganov KD, Boldin MP, Chang KJ, Baltimore D (2006): NF-kappaB-dependent induction of microRNA miR-146, an inhibitor targeted to signaling proteins of innate immune responses. Proc Natl Acad Sci U S A 103: 12481-12486.

38. Lo WL, Yu CC, Chiou GY, et al. (2011): MicroRNA-200c attenuates tumour growth and metastasis of presumptive head and neck squamous cell carcinoma stem cells. J Pathol 223: 482-495.

39. Su W, Xu M, Chen X, et al. (2015): MiR200c targets IRS1 and suppresses prostate cancer cell growth. Prostate 75: 855862.

40. Wu D, Niu X, Pan H, et al. (2016): MicroRNA-335 is downregulated in bladder cancer and inhibits cell growth, migration and invasion via targeting ROCK1. Mol Med Rep 13: 43794385 .

41. Wang Y, Wang H, Ding Y, et al. (2017): N-peptide of vMIP-II reverses paclitaxel-resistance by regulating miRNA-335 in breast cancer. Int J Oncol 51: 918-930.

42. Fu Q, Liu X, Liu Y, et al. (2015): MicroRNA-335 and -543 suppress bone metastasis in prostate cancer via targeting endothelial nitric oxide synthase. Int J Mol Med 36: 1417-1425. 